\title{
Serum Albumin Predicts Renal Dysfunction in Chronic Liver Disease
}

\author{
George Hage-Nassar ${ }^{1}$, Ahmed Shady ${ }^{1 *}$, Monnie McGee $^{2}$ and Nora V Bergasa ${ }^{1}$ \\ ${ }^{1}$ New York Medical college, USA \\ ${ }^{2}$ Southern University, Dallas, Texas, USA
}

Received: 阱 October 3, 2018; Published: 眥 October 09, 2018

*Corresponding author: Ahmed Shady, New York Medical College, Valhalla

Abbreviations: ASA: Average Serum Albumin; LDA: Linear Discriminate Analysis; CART: Classification and Regression Tree Methods; NASH: Nephropathy and Nonalcoholic Steatohepatitis

\section{Introduction}

The prevalence of renal insufficiency in patients with chronic liver disease is unknown; furthermore, no specific etiology has been demonstrated to be the predominant cause of renal insufficiency in these patients, and no factors predicting it have been identified, although particular diseases of the liver have been traditionally linked to specific forms of renal involvement. In this context however, it has been demonstrated that therapeutic paracentesis without the intravenous administration of albumin is associated with the development of hyponatremia and/or renal failure in $20 \%$ of cases [1]. Hypoalbuminemia has also been found to be a powerful indicator of increased risk of perioperative complications in elderly patients undergoing cardiac surgery, and a serum albumin less than $3.5 \mathrm{~g} / \mathrm{dl}$ was found to be the most powerful predictor of postoperative renal dysfunction in those patients [2]. Accordingly, the aim of this study was first to determine the prevalence of renal insufficiency in patients with chronic liver disease at two inner city hospitals of Central Brooklyn, New York, and to examine whether hypoalbuminemia predicts future renal insufficiency in patients with chronic liver disease.

\section{Methods}

The study was a retrospective review of available consultations and laboratory values of patients with liver disease who had been evaluated by the Hepatology Service over a period of twelve months. Chronic liver disease was defined as an increased activity of liver associated enzymes, over the six months preceding the admission at which the first consultation was provided, and/or documented chronic liver disease by prior diagnosis with a liver biopsy or by documented history, with or without impaired synthetic liver function as suggested by low serum albumin concentration, and prolonged prothrombin time. Renal insufficiency was defined as a creatinine level of $1.5 \mathrm{mg} / \mathrm{dl}$ or more; the upper limit of normal reported by the institutional clinical laboratories. Patients with renal insufficiency were divided into three groups: Group I was comprised of patients in whom renal insufficiency was diagnosed before the diagnosis of chronic liver disease. In group II no, chronologic relation could be made between the timing of diagnosis of renal disease and that of liver disease: i.e. inability to determine which disease started first (i.e. renal versus liver), and in Group III renal insufficiency developed after the diagnosis of chronic liver disease was made. In group III patients, the serum albumin concentration was reviewed over the three months period prior to the documented onset of renal insufficiency, and an average serum albumin was calculated (ASA) and assessed as a predicting factor of renal insufficiency. To determine the prevalence of renal insufficiency, all patients with chronic liver disease were included in the analysis regardless of the time of occurrence of renal insufficiency in relation to the liver disease. Patients with liver and/or renal transplant as well as those who had renal insufficiency diagnosed before the onset of liver disease (group I patients) and/or documented to be caused by non-liver related conditions, as well as patients in whom the time of onset of renal insufficiency could not be determined in relation to the liver disease (group II patients), were not included in the analysis that assessed albumin as a predicting factor for renal insufficiency. The study was approved by the Institutional Review Board.

\section{Statistical Analysis}

The data were analyzed by linear discriminate analysis (LDA) to make predictions about group membership based on the values of albumin [3] and classification and regression tree methods (CART) to find the best "cutoff" point for the Albumin level [4]. 


\section{Results}

94 patients met the inclusion criteria for chronic liver disease (Figure 1). 37 of these patients had renal insufficiency either acute with resolution or chronic persistent at any point in time in relation to their liver disease, leading to a prevalence of $39 \%$. (Figure 1). The number of patients with renal insufficiency in groups I, II and III were 3, 9 and 25, with a proportion of $8 \%, 24 \%$, and $68 \%$ respectively (Figure 2). In group III serum albumin level was evaluated during the period extending up to 3 months prior to the onset of renal insufficiency, and average serum albumin (ASA) was calculated. 22 patients ( $88 \%$ ) had an ASA of $=<2.7 \mathrm{~g} / \mathrm{dl}, 16$ patients (64\%) had an ASA $=<2.5 \mathrm{~g} / \mathrm{dl}, 9$ patients (36\%) had an ASA > $2.5 \mathrm{~g} / \mathrm{dl}$, and only 3 patients (12\%) had an ASA > $2.7 \mathrm{~g} / \mathrm{dl}$, (Figure 3 and Figure 4; group III patients). In contrast 57 (61\%) patients with chronic liver disease had no renal insufficiency at any point in time; most of these patients ( 42 i.e.74 \%) had an ASA of $>=2.7 \mathrm{~g} / \mathrm{dl}$, while only 15 patients ( $26 \%$ ) had an ASA of $=<2.7 \mathrm{~g} / \mathrm{dl}$, and $10(17$ $\%$ ) had an ASA of $=<2.5 \mathrm{~g} / \mathrm{dl}$, (Figure 3 and Figure 4; right boxes No RI). The sensitivity and specificity of an albumin of $2.7 \mathrm{~g} / \mathrm{dl}$ or less in predicting renal insufficiency was $88 \%$ and $73.6 \%$ respectively. And the positive predictive value was calculated at $59.5 \%$.

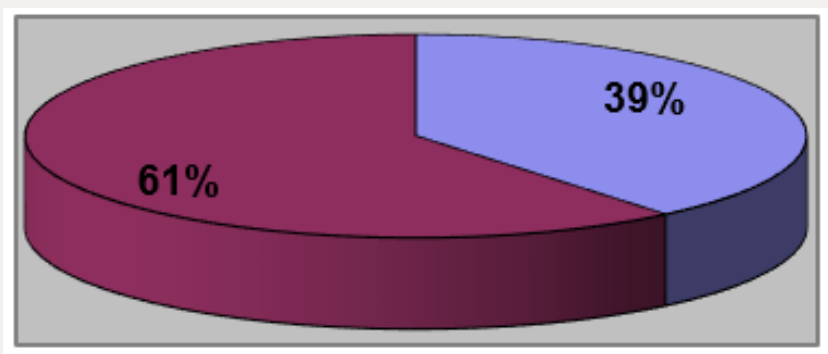

\section{口RI=37}

$\square$ No RI=57

\section{CLD patients $=94$}

Figure 1: Chart showing the total number of patients who met the criteria for CLD, and the prevalence of RI in these patients. $\mathrm{CLD}=$ Chronic Liver Disease. $\mathrm{RI}=$ Renal Insufficiency.

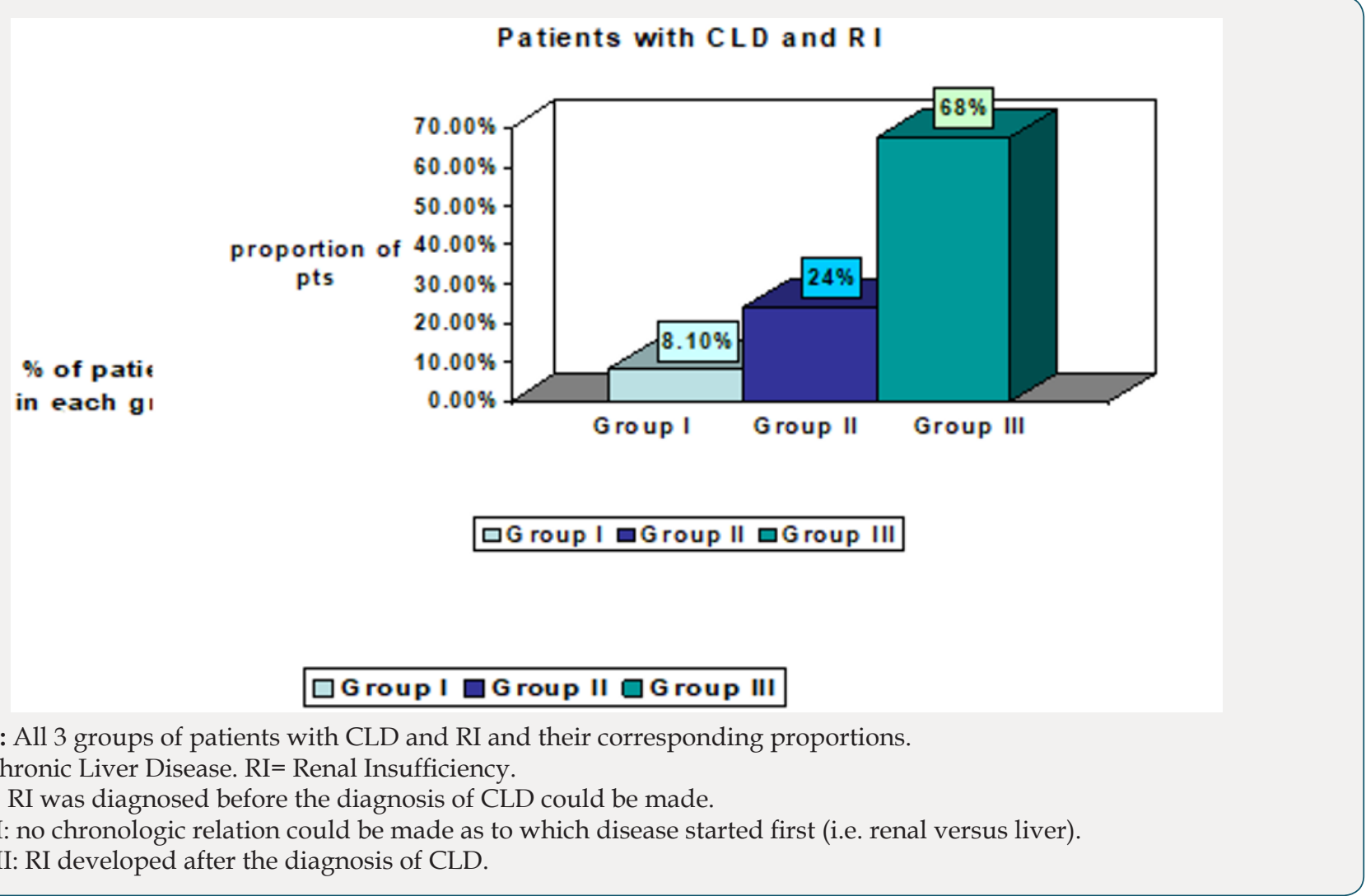




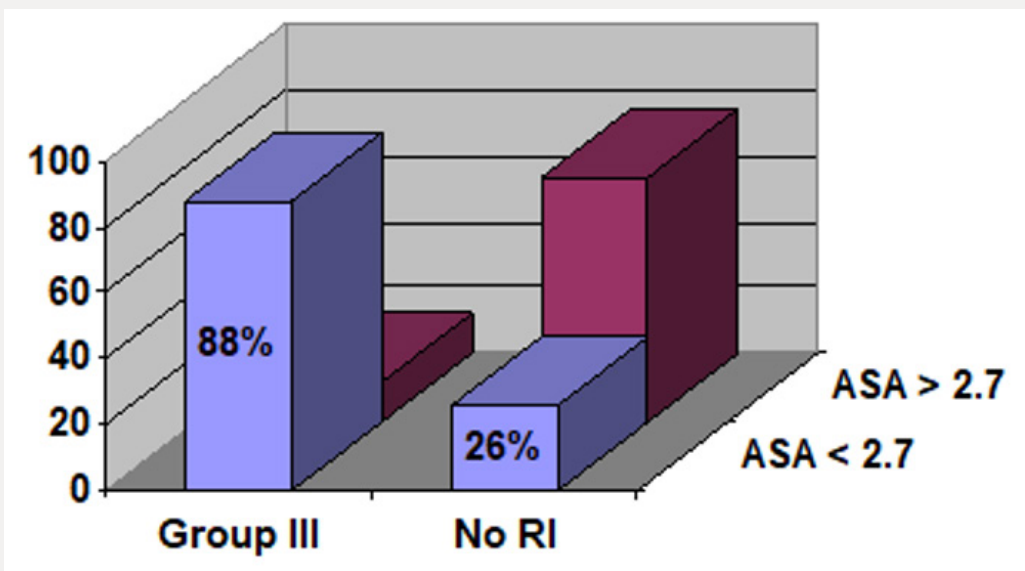

$\square \mathrm{ASA}<2.7$

$\square \mathrm{ASA}>2.7$

Figure 3: Comparison of group III patients and patients with no RI when ASA cutoff is $2.7 \mathrm{~g} / \mathrm{dl}$.RI=Renal Insufficiency. ASA=Average Serum Albumin.

Group I: RI was diagnosed before the diagnosis of CLD could be made.

Group II: no chronologic relation could be made as to which disease started first (i.e. renal versus liver).

Group III: RI developed after the diagnosis of CLD.

No RI= No renal insufficiency.

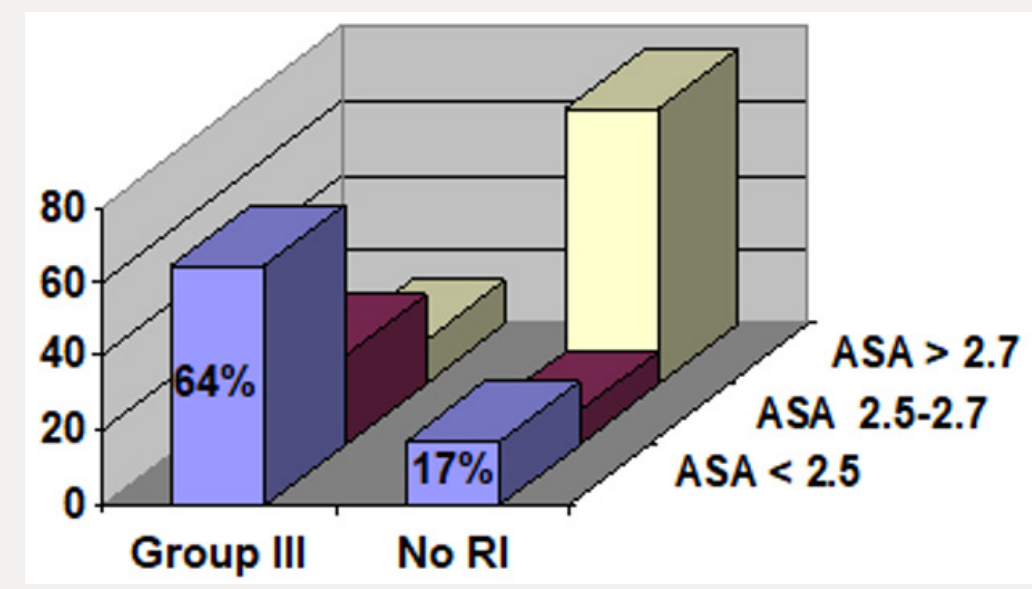

$\square$ ASA $<2.5$

$\square$ ASA $2.5-2.7$

$\square$ ASA $>2.7$

Figure 4: Comparison of group III patients and patients with no RI when ASA cutoff is $2.5 \mathrm{~g} / \mathrm{dl}$. RI=Renal Insufficiency. ASA=Average Serum Albumin.

Group I: RI was diagnosed before the diagnosis of CLD could be made.

Group II: no chronologic relation could be made as to which disease started first (i.e. renal versus liver).

Group III: RI developed after the diagnosis of CLD.

No RI= No renal insufficiency.

\section{Conclusion}

Renal insufficiency is highly prevalent in patients with chronic liver disease. Most of these patients developed their renal insufficiency after the diagnosis of chronic liver disease has been made and an average serum albumin level of $2.7 \mathrm{~g} / \mathrm{dl}$ or less, over up to three months prior to the onset of renal failure, is a sensitive predictor of the future development of renal insufficiency in this group of patients.

\section{Discussion}

Our study shows a high prevalence (39\%) of renal insufficiency in patients with chronic liver disease. Most of these patients (68\%) developed their renal insufficiency after the diagnosis of chronic liver disease was made (Group III). In this particular group the majority of patients (88\%) had an average serum albumin of 2.7 $\mathrm{g} / \mathrm{dl}$ or less over the period extending up to 3 months prior to the onset of renal insufficiency. In contrast most of the patients who had no renal insufficiency (74.1\%), had an average serum albumin of more than $2.7 \mathrm{~g} / \mathrm{dl}$. Several factors could have contributed to the high prevalence of renal insufficiency in our study. A major factor is the inclusion of patients with chronic as well as acute and subsequently resolving renal insufficiency. However even when renal insufficiency resolves, it is still a significant complication that should be accounted for in order to develop strategies to prevent 
it. One other important factor that could have contributed to the high prevalence of renal insufficiency is the inclusion of patients regardless of the chronologic relation of the renal insufficiency to the liver disease. On the other hand, creatinine clearance could not be directly measured due to the inconsistent availability of 24-hour urine creatinine samples, nor indirectly calculated due to absence of documentation of some patients' body weights and inaccuracy of lean body weights measurements in patients with ascites. Subsequently this might have underestimated the true prevalence of RI in patients with CLD, since many patients with decompensated liver disease have low muscle mass due to protein-poor diet, and diminished hepatic biosynthesis of creatine, a substrate for skeletal muscle production of creatinine, and as a result may have normal serum creatinine levels despite renal insufficiency. In fact several studies have shown that measurement of the serum creatinine level or estimating creatinine clearance or glomerular filtration rate using creatinine-based equations (e.g. Cockcroft-Gault formula) is an insensitive method for assessing renal function in ESLD patients [5-7], and ideally it has been suggested that the GFR in ESLD patients should be measured with inulin or studies using radioisotopes such as [125I] iothalamate or chromium S1-ethylene diaminetetraacetic acid or (51Cr-EDTA) clearance [8].

Identifying the etiology of renal insufficiency in patients with chronic liver disease is a major challenge and may often require renal biopsy. Hence traditionally specific renal diseases have been associated with well characterized liver pathologies; in this context, membranoproliferative glomerulonephritis (MPGN) has been described as a complication of chronic hepatitis C along with HCV related cryoglobulinemia [9,10]. Membranous and membranoproliferative glomerulonephritis (MPGN) are seen in patients with hepatitis B. IgA nephropathy is reported in association with alcoholic liver disease, and renal tubular acidosis in patients with Wilson's disease and chronic cholestasis such as that secondary to primary biliary cirrhosis [11]. Diabetic nephropathy and nonalcoholic steatohepatitis (NASH) are commonly seen in the same patient. Sarcoidosis, characterized by the presence of non-caseating granulomas, affects mostly the lungs although liver involvement is common as well as renal disease, which is mainly related to hypercalcemia [12]. Hepatic involvement is present in over $90 \%$ of patients with systemic amyloidosis, and kidney involvement is the leading cause of death, occurring in up to half of these patients [13]. Acute tubular necrosis is well established in patients with shock who also develop ischemic liver injury. Aspirin which causes renal papillary necrosis and acute tubular necrosis is also known to induce dose-related liver damage $[14,15]$. In addition, any NSAID including selective cyclo-oxygenase (COX)-2 inhibitors such as celecoxib may trigger renal insufficiency in patients with liver disease by blocking prostaglandin production [16,17].

In addition to the conditions stated above, many chronic liver disease patients particularly those with decompensated cirrhosis are exposed to therapies and clinical circumstances that place them at risk for developing renal failure including invasive diagnostic procedures, imaging studies requiring nephrotoxic contrast dyes, nephrotoxic medications, and therapies leading to volume depletion. It is well known that albumin is a negative acute phase reactant and could be falsely low in inflammatory conditions. However, by studying the average serum albumin levels over a long period of time (i.e. up to 3 months) we attempted to overcome at least in part the effect of transiently low albumin levels that could be seen temporarily during phases of inflammation. Since renal insufficiency itself adds to the morbidity and mortality of patients with liver disease [18], predicting the development of renal insufficiency in patients with chronic liver disease would not only have an important prognostic value but also a therapeutic significance if measures could be implemented to prevent this complication. Hence since hypoalbuminemia is associated with decreased effective intravascular volume, and subsequently decreased renal perfusion, having identified a cutoff serum albumin concentration $(2.7 \mathrm{~g} / \mathrm{dl})$ below which the risk of renal insufficiency becomes significantly elevated is an important finding in this study. The administration of albumin on a short term basis may be helpful to prevent renal insufficiency in patients with ascites undergoing large volume paracentesis [19], as well as in patients with hepatorenal syndrome and spontaneous bacterial peritonitis [20], thus, it is possible that albumin infusions have a similar beneficial effect on the renal function of patients with low serum albumin, regardless of the etiology of liver disease. However, the intravenous administration of albumin is not a practical longterm option. Our observation of serum albumin level as a predictor of renal insufficiency needs to be confirmed and supported by controlled, large and prospective studies that may involve the identification of a predominant etiology of renal insufficiency, and the development of new strategies to reduce the risk of this complication. These strategies could include such simple measures as discontinuing potentially nephrotoxic medications, and assuring adequate hydration, or more complicated interventions such as short-term intravenous albumin administration or the evaluation of existing pharmacological treatments like vasoconstrictors as midodrine, or terlipressin [21] that would improve systemic hemodynamics. Finally since a large proportion of patients with chronic liver disease are nowadays being managed at least in the initial stages by internists and primary care physicians, awareness of the risk of renal damage in these patients, and knowing how to predict that risk and managing it after it develops, may all need to become an integral part of the role of these physicians.

\section{References}

1. Pere Ginès, Vicente Arroyo, Joan Rodés (1989) Treatment of ascites and renal failure in cirrhosis: Bailliére's Clinical Gastroenterology 3(1): 165186.

2. Michael W Rich, Andrew J Keller, Kenneth B Schechtman, William G Marshall, Nicholas T Kouchoukos et al. (1989) Increased complications and prolonged hospital stay in elderly cardiac surgical patients with low serum albumin. The merican Journal of Cardiology 63(11): 714- 718.

3. W N, Ripley BD (2002) Modern Applied Statistics with S. Fourth: Venables ( $4^{\text {th }}$ edn) Springer, New York, USA pp. 495. 
4. Breiman, Friedman, Olshen, Stone (1984) Classification and Regression Trees: Wadsworth.

5. Pham PT, Pham PC, Wilkinson AH (2000) The kidney in liver transplantation. Clin Liver Dis 4(3): 567-90.

6. Carego L, Menon F, Angeli P (1994) Limitations of serum creatinine level and creatinine clearance as filtration markers in cirrhosis. Arch Intern Med 154(2): 201-5.

7. Sherman DS, Fish DN, Teitelbaum I (2003) Assessing renal function in cirrhotic patients: problems and pitfalls. Am J Kidney Dis 41(2): 269-78.

8. PTT PHAM, PCT PHAM, A RASTOGI, AH WILKINSON (2004) Review article: Current Management of Renal Dysfunction in the Cirrhotic Patient. 21(8): 949-961.

9. Meyers CM (2003) Hepatitis C and Renal Disease: An update. Am J Kidney Dis 42(4): 631-57.

10. Sherman DS (2003) Assessing renal function in cirrhotic patients: problems and pitfalls. Am J Kidney Dis 41(2): 269-78.

11. Wong F (2002) Liver and kidney diseases. Clin Liver Dis 6(4): 981-1011.

12. Mueller S, Boehme MW, Hofmann WJ, Stremmel W (2000) Extrapulmonary sarcoidosis primarily diagnosed in the liver. Scand J Gastroenterol 35(9): 1003-1008.

13. Schena FP, Pannarale G, Carbonara MC (1996) Clinical and therapeutic aspects of renal amyloidosis. Nephrol Dial Transplant 11(9): 63-68.

14. Seaman WE, Plotz PH, Ishak KG (1974) Aspirin-induced hepatotoxicity in patients with rheumatoid arthritis or systemic lupus erythematosus. Arthritis Rheum 17: 325.

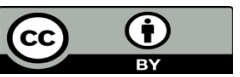

This work is licensed under Creative Commons Attribution 4.0 License

To Submit Your Article Click Here: Submit Article

DOI: $10.32474 /$ CTGH.2018.01.000120
15. Koppes GM, Arnett FC (1974) Salicylate hepatotoxicity. Postgrad Med 56:193-5.

16. Guevara M, Abecasis R, Jimenez W (2002) Effect of celecoxib on renal function in cirrhotic patients with ascites. A pilot study J Hepatol 36(1): 203.

17. Swan SK, Rudy DW, Lasseter KC, et al. (2000) Randomized evaluations of the effects of the effects of cyclooxygenase-2 inhibition on renal function in elderly persons receiving a low-salt diet: a randomized, controlled trial. Ann Intern Med 133(1): 1-9.

18. Fraley DS, Burr R, Bernardini J, Angus D, Kramer DJ, et al. (1998) Impact of acute renal failure on mortality in end-stage liver disease with or without transplantation. Kidney Int 54(2): 518-24.

19. Luca A, Garcia-Pagan JC, Bosch J, et al. (1995) Beneficial effects of intravenous albumin infusion on the hemodynamic and humoral changes after total paracentesis. Hepatology 22(3): 753-758.

20. Sort P, Navasa M, Arroyo V, Aldeguer X, Planas R, et al. (1999) Effect of intravenous albumin on renal impairment and mortality in patients with cirrhosis and spontaneous bacterial peritonitis. Rodes J N Engl J Med 341(6): 403-9.

21. Alessandria C, Venon WD, Marzano A, et al. (2002) Renal failure in cirrhotic patients: role of terlipressin in clinical approach to hepatorenal syndrome type 2. Eur J Gastroenterol Hepatol 14(12): 1363-8.

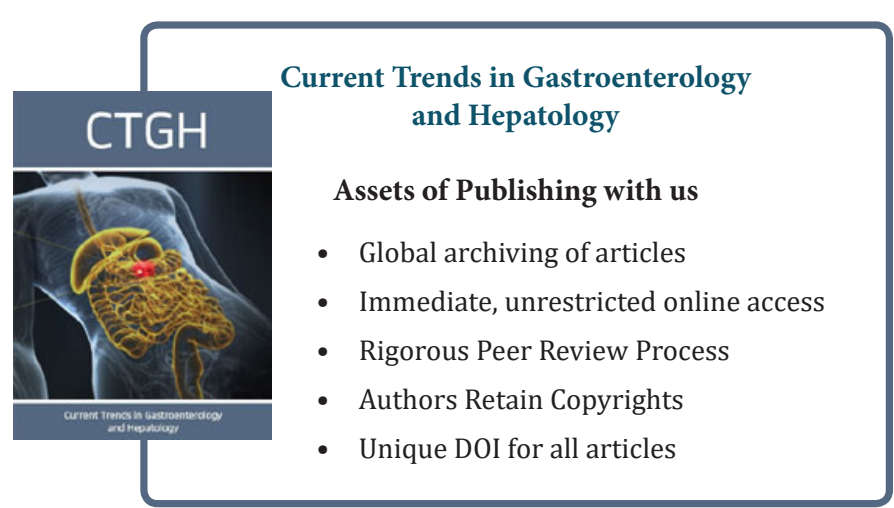

